Pacific

Journal of

Mathematics

EXISTENCE OF SINGULAR POSITIVE SOLUTIONS FOR SOME SEMILINEAR ELLIPTIC EQUATIONS

Zongming Guo, Dong Ye And Feng Zhou 


\title{
EXISTENCE OF SINGULAR POSITIVE SOLUTIONS FOR SOME SEMILINEAR ELLIPTIC EQUATIONS
}

\author{
Zongming GuO, Dong Ye And Feng Zhou
}

\begin{abstract}
We study positive solutions of an equation with singular nonlinearities. The equation arises in the study of equilibrium states of thin films. Under weak assumptions on the nonlinearity, we show that for $N \geq \mathbf{3}$ there exists a family of radial solutions $\left\{u_{\alpha}\right\}_{\alpha>0}$ with $u_{\alpha}(0)=\alpha$ and each of them is oscillatory in $(0, \infty)$. We obtain then a singular radial solution in $(0, \infty)$ by taking the limit $\alpha \rightarrow 0$. Meanwhile, using the solutions obtained in $(0, \infty)$, we show some existence results for the corresponding Neumann eigenvalue problem on a ball.
\end{abstract}

\section{Introduction}

This paper concerns mainly the structure of positive radial solutions of the semilinear elliptic equation in $\mathbb{R}^{N}$ given by

$$
\Delta u=f(u),
$$

where $f \in C^{1}(0, \infty)$ is a given nonlinear function which tends to $\infty$ at $0^{+}$. A class of typical examples is $f(u)=u^{-p}-\mu_{1} u^{-q}-\mu_{2}$ with constants $p>\max (q, 0)$. This kind of semilinear equation appears in several applications in mechanics and physics. For example, equations of the type

$$
u_{t}=-\nabla \cdot[h(u) \nabla \Delta u]-\nabla \cdot[g(u) \nabla u]
$$

have been used to model the dynamics of thin films for viscous liquids, where $z=u(x, y, t)$ is the height of the air/liquid interface. The zero set

$$
\Sigma=\left\{x \in \Omega: \lim _{r \rightarrow 0^{+}} \frac{1}{\left|B_{r}(x)\right|} \int_{B_{r}(x)} u(y) d y \text { exists and is equal to } 0\right\},
$$

with $B_{r}(x)$ being the ball with center at $x$ and radius $r$, is called set of ruptures, which plays a very important role in the study of thin films. The coefficient $h(u)$ is determined by surface tension effects, and the coefficient $g(u)$ can model additional

MSC2000: 35J60, 35B45, 35B05.

Keywords: elliptic equation with singular nonlinearity, rupture solutions, oscillation.

Guo is partially supported by NSFC (Number 10571022). Zhou is partially supported by NNSF Number 10671071 of China and the basic research project (973, Number 2006CB805902) of China. 
forces. Polynomials are often chosen for $h$ and $g$. For example, in the van der Waals model we may choose $h(u)=u^{3}$ and $g(u)=u^{m}-\kappa u^{n}$, with suitable constants $\kappa, m, n$. If we consider the steady states of Equation (1-2), we see that $u$ satisfies

$$
h(u) \nabla \Delta u+g(u) \nabla u=\mathscr{C} \quad \text { in } \Omega,
$$

where $\mathscr{C}$ is a constant vector. Assuming $\mathscr{C}=0_{\mathbb{R}^{N}}$, let $v=(2-m)^{1 /(3-m)} u$; then a direct calculation yields that

$$
\Delta v=v^{m-2}-\frac{\kappa(2-m)^{(3-n) /(3-m)}}{(2-n)} v^{n-2}-C_{0} \quad \text { in } \Omega .
$$

Some detailed physics background is found in [Bertozzi et al. 2001; Bertozzi and Pugh 1998; 2000; Burelbach et al. 1988; Hwang et al. 1997; Jones and Küpper 1986; Joseph and Lundgren 1972/73; Laugesen and Pugh 2000a; 2000b]. Some recent mathematical analysis is found in [Grün 2004; Jiang and Lin 2004; Jiang and Ni 2007; Li et al. 2005; Slepčev and Pugh 2005].

In this paper, we study of the radial solutions of Equation (1-1); hence we always write it in the radial version

$$
u^{\prime \prime}+\frac{N-1}{r} u^{\prime}=f(u) \text { for } r=\|x\|=\sqrt{x_{1}^{2}+x_{2}^{2} \cdots+x_{N}^{2}} \in[0, \infty) .
$$

Here $f$ satisfies two general conditions:

Condition 1.1. (i) $f$ has a single zero $t_{0}$ in $(0, \infty)$ satisfying $f^{\prime}\left(t_{0}\right)<0$,

(ii) $f$ is nonincreasing near 0 , and $\lim _{t \rightarrow 0^{+}} f(t)=\infty$.

Remark 1.2. Here we do not need any monotonicity assumption at infinity for $f$, and no additional condition on the primitive of $f$ is required.

As a very special case of our main result, let $f(u)=u^{-p}-\mu_{1} u^{-q}-\mu_{2}$ for any constants $p \geq 1>q>0$ and $\mu_{2}>0$. We will show (see Theorem 2.9 and Remark 2.10) in dimension $N \geq 3$ that if $\alpha>0$ with $\alpha \neq t_{0}$, where $t_{0}$ is the unique zero of $f$, then Equation (1-3) has a global solution $u_{\alpha}$ with $u_{\alpha}(0)=\alpha$. Moreover, $u_{\alpha}$ is infinitely oscillatory around the constant $t_{0}$. That is, there exists a sequence $\rho_{\alpha}^{n}$ tending to $\infty$ such that $u_{\alpha}\left(\rho_{\alpha}^{n}\right)=t_{0}$ for $n \in \mathbb{N}$ and such that $u_{\alpha}-t_{0}$ changes sign at $\rho_{\alpha}^{n}$. We present a uniform approach for $N \geq 3$, since we have different behaviors in lower dimensions $N=1,2$, for which some special results will be given in the appendix.

Meanwhile, we show that when $N \geq 3$, Equation (1-3) has a singular (or rupture) solution $u_{0}$ which is also oscillatory; see Theorem 3.1. More precisely, $u_{0}$ is a continuous radial function in $\mathbb{R}^{N}$ and is a weak solution of (1-1) in $\mathbb{R}^{N}$ such that $u_{0}(0)=0$.

Jiang and Ni [2007] have shown the existence and uniqueness of the radial rupture solution when $f(u)=u^{-p}-\mu_{2}$ in $\mathbb{R}^{N}$ with $p, \mu_{2}>0$ and $N \geq 2$. Their proof 
for the existence of rupture solution used more-involved ODE techniques and is very different from our method.

Using the entire solutions, meaning those defined on the whole space, we obtain easily the structure of radial solutions for the eigenvalue Neumann problem

$$
\Delta u=\lambda f(u) \quad \text { in } B, \quad \frac{\partial u}{\partial v}=0 \quad \text { on } \partial B,
$$

where $\lambda>0, f$ satisfies Condition 1.1, and $B=B_{1}=\left\{x \in \mathbb{R}^{N}:\|x\|<1\right\}$ is the unit ball of $\mathbb{R}^{N}$.

When $f$ is positive, for example, if $f(u)=u^{-p}$ with $p>0$, then is clear that any radial solution is increasing with the radius $r$. Guo and Wei [2007] give some interesting necessary and sufficient conditions to ensure the radial symmetry of entire solutions; however we do know that nonradial solutions exist, for example, when $N=2$ and $p=3$. For entire radial solution of $\Delta u=K(r) u^{-p}$ with bounded, positive and, nonincreasing coefficient function $K$ and $p>0$, the asymptotic behavior of $u$ near $\infty$ was established in [Guo et al. 2006].

\section{Existence and oscillatory properties of solutions of (1-3)}

We first remark that any classical radial solution of (1-3) solves the initial value problem

$$
u^{\prime \prime}+\frac{N-1}{r} u^{\prime}=f(u) \quad \text { in } \mathbb{R}_{+}, \quad \text { with } u(0)=\alpha>0 \quad \text { and } \quad u^{\prime}(0)=0 .
$$

In this section, we assume always $N \geq 3$. We claim then for any $\alpha \in\left(0, t_{0}\right)$ that the solution $u_{\alpha}(r)$ of (2-1) is oscillatory around the unique zero of $f$, and $u_{\alpha}(r)>0$ for all $r>0$. Throughout the paper, the symbols $C$ and $C_{i}$ denote positive constants, though they may change from one line to another.

Theorem 2.1. Suppose that $f \in C^{1}(0, \infty)$ satisfies only Condition 1.1(i). Then for each $\alpha \in\left(0, t_{0}\right),(2-1)$ has a unique positive solution $u_{\alpha}$, which oscillates around the constant $t_{0}$, that is, there is an increasing positive sequence $\left\{r_{\alpha}^{n}\right\}$ such that $\left\{r>0, u_{\alpha}^{\prime}(r)=0\right\}=\left\{r_{\alpha}^{n}\right\}$ and $\lim _{n \rightarrow \infty} r_{\alpha}^{n}=\infty$. Also the $r_{\alpha}^{2 i+1}$ are local maxima of $u_{\alpha}$ with $u_{\alpha}\left(r_{\alpha}^{2 i+1}\right)>t_{0}$ for any $i \in \mathbb{N}$, while the $r_{\alpha}^{2 i}$ are local minima with $u_{\alpha}\left(r_{\alpha}^{i}\right)<t_{0}$. Proof. By standard ODE theory, Equation (2-1) has a unique solution $u_{\alpha}$ near $r=0$ for any fixed $0<\alpha<t_{0}$, and $u_{\alpha}$ can be extended whenever it exists. For simplicity, we will omit the index $\alpha$. Thus

$$
\left(r^{N-1} u^{\prime}\right)^{\prime}=r^{N-1} f(u) .
$$

Condition 1.1(i) implies that $f(t)>0$ in $\left(0, t_{0}\right)$ and $f(t)<0$ for any $t>t_{0}$, so $u$ is increasing near $r=0$ as $\alpha \in\left(0, t_{0}\right)$. We want to prove that the solution exists on all of $\mathbb{R}_{+}$. 
Assume that $u$ exists in $\left[0, r_{\max }\right)$. Define

$$
F(t)=\int_{t_{0}}^{t} f(s) d s
$$

Multiplying (2-1) by $u^{\prime}$, we find

$$
\left(\frac{u^{\prime 2}}{2}-F(u)\right)^{\prime}(r)=-\frac{(N-1)}{r} u^{\prime}(r)^{2} \leq 0 \quad \text { for all } r \in\left[0, r_{\max }\right) .
$$

Therefore the function $u^{\prime}(r)^{2} / 2-F(u(r))$ is decreasing in $\left[0, r_{\max }\right)$, and so

$$
\frac{1}{2} u^{\prime}(r)^{2}-F(u(r)) \leq-F(\alpha) .
$$

Hence $F(u(r)) \geq F(\alpha)$, and we get readily $u(r) \geq \alpha$ in $\left[0, r_{\max }\right)$ by the variation of $F$. It remains to show that $u$ cannot tend to infinity at a finite value of $r$.

We now claim $u$ has a turning point. That is, there exists an $r^{1}>0$ such that $u^{\prime}\left(r^{1}\right)=0$ and $u^{\prime \prime}\left(r^{1}\right)<0$. In fact, let $r^{1}=\inf \left\{r>0: u^{\prime}(r)=0\right\}$. If $r^{1}<\infty$ exists, then, since $r^{N-1} u^{\prime}$ increases until $u$ reaches $t_{0}$, it is clear that $u\left(r^{1}\right)>t_{0}$. Hence $u^{\prime \prime}\left(r^{1}\right)<0$ by Equation (2-1). If $r^{1}$ does not exist, $u$ is increasing in [0, $\left.r_{\max }\right)$. Since $f$ is negative in $\left(t_{0}, \infty\right)$, we get $0 \leq r^{N-1} u^{\prime} \leq C$ in $\left(0, r_{\max }\right)$. Using $N \geq 3$, we find that $\lim _{r \rightarrow r_{\max }} u(r)=\beta \in(0, \infty)$ exists. Hence $r_{\max }=\infty$. Furthermore, if $f(\beta)>0$, we have then $\Delta u \geq C>0$ for $r$ big enough, and we easily get $u(r) \geq C_{1} r^{2}-C_{2}$, which contradicts $\beta \in \mathbb{R}$. Similarly, we cannot have $f(\beta)<0$, so we must have $\beta=t_{0}$. But this is also impossible by Lemma 2.2 below, whose proof we postpone. Our claim is proved.

Lemma 2.2. Suppose that an entire solution $u_{\alpha}$ of (2-1) exists with $f$ satisfying (i) and $N \geq 3$. Then there is no $r_{0}>0$ such that $u_{\alpha}>t_{0}$ or $u_{\alpha}<t_{0}$ for all $r \geq r_{0}$.

Moreover, the monotonicity of $u^{\prime 2} / 2-F(u)$ and the variation of $F$ show that $u(r) \leq u\left(r^{1}\right)$ for any $r \geq r^{1}$. In conclusion, the solution $u$ exists in $\mathbb{R}_{+}$, and $u(r) \in\left[\alpha, u\left(r^{1}\right)\right]$. The oscillatory property of $u$ is insured by Lemma 2.2, which then implies there is an increasing sequence $\left\{r^{i}\right\}$ such that $\lim _{i \rightarrow \infty} r^{i}=\infty$ and $u^{\prime}\left(r^{i}\right)=0$.

We see that if $r_{*}$ is a local minimum of $u$, then $u^{\prime \prime}\left(r_{*}\right) \geq 0$ and $u^{\prime}\left(r_{*}\right)=0$. Thus $u\left(r_{*}\right)<t_{0}$ by Equation (2-1), since we cannot have $u\left(r_{*}\right)=t_{0}$. On the other hand, any local maximum $r^{*}$ of $u$ satisfies $u\left(r^{*}\right)>t_{0}$. Recall that $r^{1}$ realizes a local maximum of $u$; so $r^{2 i+1}$ are local maxima, while $r^{2 i}$ are local minima.

Proof of Lemma 2.2. Suppose that there exists an $r_{0}>0$ such that $u>t_{0}$ for all $r \geq r_{0}$, and so $\Delta u \leq 0$ for $r \geq r_{0}$. As above, we can show that $u$ is uniformly bounded and $u(r)$ tends to $t_{0}$ at $\infty$. Set $v=u-t_{0}$. Then $v>0$ in $\left[r_{0}, \infty\right)$, and $v$ satisfies the differential equation $v^{\prime \prime}+((N-1) / r) v^{\prime}-f^{\prime}(\xi) v=0$ with $\xi(r) \in\left(t_{0}, u(r)\right)$. By $\lim _{r \rightarrow \infty} u(r)=t_{0}$ and Condition 1.1(i), there exist constants $C_{0}>0$ and $r_{1} \geq r_{0}$ such that $-\Delta v \geq C_{0} v$ in $\mathbb{R}^{N} \backslash B_{r_{1}}$. 
However, this is impossible. Indeed, fix $R>r_{1}$ large enough so that $\lambda_{1}(-\Delta)$ is less than $C_{0} / 2$ in $H_{0}^{1}\left(A_{R}\right)$, where $A_{R}=B_{R} \backslash \bar{B}_{r_{1}}$. Letting $\Phi_{1} \in H_{0}^{1}\left(A_{R}\right)$ be a corresponding eigenfunction such that $\Phi_{1}>0$ in $A_{R}$, we get

$$
\int_{A_{R}}(-\Delta v) \Phi_{1} d x \geq \int_{A_{R}} C_{0} v \Phi_{1} d x .
$$

This yields, because $\partial_{\nu} \Phi_{1}<0$ on $\partial A_{R}$, that

$$
0<\left(C_{0}-\lambda_{1}\right) \int_{A_{R}} v \Phi_{1} d x \leq \int_{\partial A_{R}} \frac{\partial \Phi_{1}}{\partial v} v d s<0,
$$

and so the hypothesis is false. Similarly, we can prove that these is no $r_{0}>0$ such that $u<t_{0}$ in $\left[r_{0}, \infty\right)$.

Remark 2.3. By (2-4), it is easy to show that $u_{\alpha}\left(r_{\alpha}^{2 i+1}\right)$ is a decreasing sequence and that $u_{\alpha}\left(r_{\alpha}^{2 i}\right)$ is increasing.

Therefore $\lim _{i \rightarrow \infty} u_{\alpha}\left(r_{\alpha}^{2 i+1}\right)=\bar{t}$ and $\lim _{i \rightarrow \infty} u_{\alpha}\left(r_{\alpha}^{2 i}\right)=\underline{t}$ exist. In fact, $\bar{t}=\underline{t}=t_{0}$.

Theorem 2.4. Suppose that $f \in C^{1}(0, \infty)$ satisfies Condition 1.1(i). Then any oscillating solution $u_{\alpha}$ of (2-1) satisfies $\lim _{r \rightarrow \infty} u_{\alpha}(r)=t_{0}$.

Proof. We omit the fixed index $\alpha$. By (2-4) and the boundedness of $u$,

$$
\lim _{r \rightarrow \infty}\left(\frac{1}{2} u^{\prime}(r)^{2}-F(u(r))\right)=\ell
$$

for some constant $\ell \in \mathbb{R}$. It suffices to prove that $\ell=0=-F\left(t_{0}\right)$. Indeed, letting $\left\{r^{k}\right\}$ be the sequence of local extremum points of $u$, we have $\lim _{k \rightarrow \infty} F\left(u\left(r^{k}\right)\right)=0$, and hence $\lim _{k \rightarrow \infty} u\left(r^{k}\right)=t_{0}$. Then $\lim _{r \rightarrow \infty} u(r)=t_{0}$ by Remark 2.3.

It is clear that $\ell \geq 0$ since $F \leq 0$ in $\mathbb{R}_{+}$. Suppose by contradiction that $\ell>0$, so $\bar{t}>\underline{t}$. We claim then

$$
\text { there exists a } C>0 \text { such that } r^{k+1}-r^{k} \leq C<\infty \text { for all } k \in \mathbb{N}^{*} \text {. }
$$

Let $s_{k} \in\left(r^{k}, r^{k+1}\right)$ be the unique point with $u\left(s_{k}\right)=t_{0}$. Using (2-4) again, we have $\frac{1}{2} u^{\prime}\left(s_{k}\right)^{2} \geq \ell+F\left(t_{0}\right)$, that is, $\left|u^{\prime}\left(s_{k}\right)\right| \geq \sqrt{2 \ell}>0$. On the other hand, we know that $\alpha \leq u \leq u\left(r^{1}\right)$ for all $r \in\left[r^{1}, \infty\right)$. Therefore $f(u)$ is uniformly bounded in $\left[r^{1}, \infty\right)$. Because $F \leq 0, \frac{1}{2} u^{\prime}(r)^{2} \leq F(u(r))-F\left(u\left(r^{1}\right)\right) \leq-F\left(u\left(r^{1}\right)\right)$ for all $r \geq r^{1}$; hence $u^{\prime}(r)$ is uniformly bounded in $\left[r^{1}, \infty\right)$. By (2-1), we see that $u^{\prime \prime}(r)$ is uniformly bounded in $\left[r^{1}, \infty\right)$. There is an $\eta>0$ such that $\left|u^{\prime}(r)\right| \geq \sqrt{\ell}>0$ for all $r \in\left[s_{k}-\eta, s_{k}+\eta\right]$ with $k \geq 1$.

Fixing $k \geq 1$, we may assume without loss of generality that $u$ is decreasing in $\left[r^{k}, r^{k+1}\right]$. In the interval $\left[r^{k}, s_{k}-\eta\right]$ (if $r^{k}<s_{k}-\eta$ ), we have $u(r) \geq t_{0}+\sqrt{\ell} \eta$, which implies that

$$
\int_{r^{k}}^{s_{k}-\eta} f(u(r)) d r \leq-C_{1}\left(s_{k}-\eta-r^{k}\right),
$$


where $C_{1}=-\max _{\left[t_{0}+\sqrt{\ell} \eta, u\left(r^{1}\right)\right]} f(t)>0$. Also, since $u^{\prime} \leq 0$ in $\left[r^{k}, s_{k}-\eta\right]$, we see

$$
\int_{r^{k}}^{s_{k}-\eta}\left(u^{\prime \prime}(r)+\frac{N-1}{r} u^{\prime}(r)\right) d r \geq u^{\prime}\left(s_{k}-\eta\right)+\frac{N-1}{r^{1}} \int_{r^{k}}^{s_{k}-\eta} u^{\prime}(r) d r \geq-C_{2} .
$$

Combining the last two results and Equation (2-1), we get $s_{k}-\eta-r^{k} \leq C$ for any $k \geq 1$. By the same, $r^{k+1}-s_{k}-\eta \leq C$ for $k \geq 1$. Finally we get $r^{k+1}-r^{k} \leq 2 \eta+C$, which verifies the claim (2-7). We now finish the proof by following the argument in [Jiang and Ni 2007]. Because

$$
\int_{r^{k}}^{r^{k+1}} \frac{d r}{r} \leq \ln \left(\frac{r^{k}+C}{r^{k}}\right) \leq \frac{C}{r^{k}} \leq \frac{C_{3}}{r^{k+1}}
$$

and $\left|u\left(r^{k+1}\right)-u\left(r^{k}\right)\right| \geq \bar{t}-\underline{t}=\delta>0$, we obtain

$$
\int_{r^{k}}^{r^{k+1}} \frac{u^{\prime}(r)^{2}}{r} d r \geq \frac{1}{r^{k+1}} \int_{r^{k}}^{r^{k+1}} u^{\prime}(r)^{2} d r \geq \frac{\delta^{2}}{C r^{k+1}} \geq C_{4} \int_{r^{k}}^{r^{k+1}} \frac{d r}{r} .
$$

However, $u^{\prime}(r)^{2} / r$ is integrable on $\left[r^{1}, \infty\right)$ by (2-4). This contradicts the above estimate, and so we are done.

Under another assumption on $f$, we get also the oscillation between two radial solutions with different initial data.

Proposition 2.5. Suppose that $f$ satisfies Condition 1.1(i) and $f^{\prime}$ is negative in $(0, \infty)$. Let $t_{0}>\alpha$ and $\beta>0$ be different constants. Then the graph of $u_{\alpha}$ oscillates around that of $u_{\beta}$.

Proof. Suppose the contrary. Without losing generality, we may assume that $u_{\alpha}(r) \geq u_{\beta}(r)$ for $r \in\left[r_{1}, \infty\right)$ with some $r_{1}>0$. Then setting $w=u_{\alpha}-u_{\beta}$, we find $w \geq 0$ in $\left[r_{1}, \infty\right)$, and $w$ satisfies $\Delta w+f^{\prime}(\zeta) w=0$ in $\mathbb{R}^{N} \backslash B_{r_{1}}$, where $\zeta \in\left(u_{\beta}, u_{\alpha}\right)$. The boundedness of $u_{\alpha}$ and $u_{\beta}$ given by Theorem 2.1 means that there exists a $C>0$ such that $-f^{\prime}(\zeta) \geq C$. Again the eigenvalue argument as in the proof of Lemma 2.2 shows that it is impossible.

Define $Z(\alpha, \beta)$ to be the first zero of $u_{\alpha}-u_{\beta}$, where $\alpha>\beta>0$. Then by Proposition $2.5, Z(\alpha, \beta)<\infty$ for all $t_{0}>\alpha>\beta>0$. If $f$ is also convex in $\mathbb{R}_{+}$ (for example, $f(t)=u^{-p}-C$ with $p, C>0$ ), then $Z(\alpha, \beta)$ has the following monotonicity property.

Corollary 2.6. If $f$ is decreasing, convex in $\mathbb{R}_{+}$, and satisfies Condition 1.1(i), then for any $t_{0}>\alpha>\beta>\gamma>0$, we have $\min \{Z(\alpha, \beta), Z(\alpha, \gamma)\} \geq Z(\beta, \gamma)$.

Proof. Suppose for contradiction that $Z(\beta, \gamma)>Z(\alpha, \beta)$. Setting $z_{1}=u_{\alpha}-u_{\beta}$, we have $z_{1}>0$ in $[0, Z(\alpha, \beta))$ and $\Delta z_{1}+k_{1}(x) z_{1}=0$, where

$$
k_{1}(x):=-\frac{f\left(u_{\alpha}\right)-f\left(u_{\beta}\right)}{u_{\alpha}-u_{\beta}} \leq-f^{\prime}\left(u_{\beta}\right) \quad \text { in }\|x\|<Z(\alpha, \beta) .
$$


Next, setting $z_{2}=u_{\beta}-u_{\gamma}$, we have $z_{2}>0$ in $[0, Z(\beta, \gamma))$ and $\Delta z_{2}+k_{2}(x) z_{2}=0$, where

$$
k_{2}(x):=-\frac{f\left(u_{\beta}\right)-f\left(u_{\gamma}\right)}{u_{\beta}-u_{\gamma}} \geq-f^{\prime}\left(u_{\beta}\right) \geq k_{1}(x) \text { in }\|x\|<Z(\beta, \gamma) .
$$

Since $z_{2}>0$ and $-\Delta z_{1}-k_{2}(x) z_{1} \leq 0$ in $B_{Z(\alpha, \beta)}$, multiplying by $z_{2}$ and integrating by parts, we use $z_{1}^{\prime}(Z(\alpha, \beta))<0$ and a standard ODE argument to get

$$
0 \geq \int_{B_{Z(\alpha, \beta)}}\left(-\Delta z_{1}-k_{2}(x) z_{1}\right) z_{2} d x=-\int_{\partial B_{Z(\alpha, \beta)}} \frac{\partial z_{1}}{\partial r} z_{2} d \sigma>0 .
$$

This is of course absurd, and so we get $Z(\beta, \gamma) \leq Z(\alpha, \beta)$. This automatically gives $Z(\alpha, \gamma) \geq Z(\beta, \gamma)$.

Remark 2.7. By the same proof, we have that if $f$ is strictly convex, the inequality is strict in Corollary 2.6.

Remark 2.8. Becausee we are interested in rupture solutions, we show only the "small" $\alpha$ case. For the case $\alpha>t_{0}$ with suitable assumptions on $\alpha$, we can prove without difficulty the analogues of the global existence and oscillatory results of Theorem 2.1. We leave the proof to interested readers.

Theorem 2.9. Assume that $f \in C^{1}(0, \infty)$ satisfies Condition 1.1(i). Let $F$ be defined by (2-3), and suppose $\alpha^{*}=\sup \left\{s>t_{0}: F(s) \geq \lim _{t \rightarrow 0^{+}} F(t)\right\}$. Then $u_{\alpha}$ exists globally in $\mathbb{R}_{+}$if $t_{0}<\alpha<\alpha^{*}$, and there is an increasing sequence $\left\{r_{\alpha}^{i}\right\}$ such that for any $i \in \mathbb{N}, r_{\alpha}^{2 i+1}$ is a local minimum of $u_{\alpha}$ with $u_{\alpha}\left(r_{\alpha}^{2 i+1}\right)<t_{0}$, while $r_{\alpha}^{2 i}$ is a local maximum of $u_{\alpha}$ with $u_{\alpha}\left(r_{\alpha}^{i}\right)>t_{0}$. Also, $\lim _{r \rightarrow \infty} u_{\alpha}(r)=t_{0}$.

Remark 2.10. In particular, if $\lim _{t \rightarrow 0^{+}} F(t)=-\infty$, we have $\alpha^{*}=\infty$. Hence for any $\alpha>0$ with $\alpha \neq t_{0}$, the solution $u_{\alpha}$ to (2-1) oscillates infinitely around $t_{0}$ and converges to $t_{0}$ as $r \rightarrow \infty$. Furthermore, Proposition 2.5 and Corollary 2.6 hold if we change $t_{0}$ to $\alpha^{*}$.

\section{Existence of a singular solution}

Now we prove the existence of an entire rupture solution of (1-1) under Condition 1.1. Recall that $N \geq 3$.

Theorem 3.1. Given $f$ satisfying Condition 1.1, there is a singular radial solution $u_{0}(r)$ of (1-1) such that $u_{0} \in C\left(\mathbb{R}^{N}\right), u_{0}(0)=0, u_{0}(r)>0$ for $r \in(0, \infty)$, and $f\left(u_{0}\right) \in L_{\text {loc }}^{1}\left(\mathbb{R}^{N}\right)$. Also any singular radial solution of (1-1) oscillates around $t_{0}$ and converges to $t_{0}$ as $r \rightarrow \infty$.

Proof. The idea is to get a singular solution $u_{0}$ by taking limit of solutions $u_{\alpha}$ as $\alpha$ tends to $0^{+}$. A key argument is to show that for any $r>0, u_{\alpha}(r)$ is uniformly bounded and distinct from 0 for $\alpha>0$ small enough. 
Fix $t_{1} \in\left(0, t_{0}\right]$ such that $f$ is nonincreasing in $\left(0, t_{1}\right]$. For any $\alpha \in\left(0, t_{1}\right)$, let $r_{1}(\alpha)=\inf \left\{r>0, u_{\alpha}(r)=t_{1}\right\}$; such $r_{1}$ exists due to the oscillation of $u_{\alpha}$. Since $f>0$ in $\left(0, t_{1}\right)$ and

$$
r^{N-1} u_{\alpha}^{\prime}(r)=\int_{0}^{r} f\left(u_{\alpha}(s)\right) s^{N-1} d s,
$$

we get that $u_{\alpha}$ is increasing in $\left[0, r_{1}\right]$ from $\alpha$ to $t_{1}$; hence $f\left(u_{\alpha}(r)\right)$ is decreasing in $\left[0, r_{1}\right]$. Therefore we have $u_{\alpha}^{\prime}(r) \geq f\left(u_{\alpha}(r)\right) r / N$ for all $r \in\left[0, r_{1}\right]$. Now define $G(t)=\int_{0}^{t}(1 / f(s)) d s$ for $t<t_{0}$. The latest estimate for $u_{\alpha}^{\prime}(r)$ then implies

$$
G\left(u_{\alpha}(r)\right) \geq \frac{r^{2}}{2 N}+G(\alpha) \geq \frac{r^{2}}{2 N} \text { for all } r \in\left[0, r_{1}\right] .
$$

Noting that $G$ is increasing in $\left[0, t_{0}\right)$, we conclude that

$$
u_{\alpha}(r) \geq G^{-1}\left(\frac{r^{2}}{2 N}\right) \quad \text { for any } \alpha \in\left(0, t_{1}\right) \text { and } r \in\left[0, r_{1}(\alpha)\right) \text {. }
$$

Now we will prove that there exists a $C_{1}>0$ such that $r_{1}(\alpha) \geq C_{1}$ for small $\alpha$. Combining (3-1), (3-2), and the monotonicity of $f$ in $\left(0, t_{1}\right]$, we have

$$
u_{\alpha}^{\prime}(r) \leq r^{1-N} \int_{0}^{r} f \circ G^{-1}\left(\frac{s^{2}}{2 N}\right) s^{N-1} d s \quad \text { for } r \leq r_{1} .
$$

Integrating from 0 to $r_{1}$, we get

$$
\begin{aligned}
t_{1}-\alpha & \leq \int_{0}^{r_{1}} r^{1-N} \int_{0}^{r} f \circ G^{-1}\left(\frac{s^{2}}{2 N}\right) s^{N-1} d s d r \\
& =\int_{0}^{r_{1}} f \circ G^{-1}\left(\frac{s^{2}}{2 N}\right) s^{N-1} \int_{s}^{r_{1}} r^{1-N} d r d s \\
& \leq \frac{1}{N-2} \int_{0}^{r_{1}} f \circ G^{-1}\left(\frac{s^{2}}{2 N}\right) s d s \\
& =\frac{N}{N-2} \int_{0}^{r_{1}^{2} /(2 N)} f \circ G^{-1}(\sigma) d \sigma=\frac{N}{N-2} G^{-1}\left(\frac{r_{1}^{2}}{2 N}\right) .
\end{aligned}
$$

The last equality is obtained by the changes of variable $t=G^{-1}(\sigma)$ and $G^{\prime}=f^{-1}$. For all $0<\alpha<t_{1} / 2$, we obtain finally

$$
\frac{r_{1}^{2}}{2 N} \geq G\left(\frac{(N-2) t_{1}}{2 N}\right) \geq G\left(\frac{t_{1}}{6}\right)>0,
$$

which means that $r_{1}(\alpha)$ is uniformly bounded from below for $\alpha>0$ small enough. The inequalities (3-2), (3-3), and (3-4) are key arguments for our proof, and they will help us prove some uniform estimates.

We first prove the local existence of a singular solution and then extend it to a global solution. Noting that $u_{\alpha}$ is increasing as it approaches $t_{1}$, we have by (3-2) 
and (3-4) that

$$
G^{-1}\left(\frac{\delta^{2}}{2 N}\right) \leq u_{\alpha}(r) \leq t_{1} \quad \text { for all } \alpha<\delta \leq r \leq \sqrt{2 N C_{0}}=: r_{*},
$$

where $C_{0}=G\left(t_{1} / 6\right)$.

Using standard elliptic theory and the diagonal process, we obtain a subsequence such that $u_{\alpha_{n}}$ converges in $C^{1}$ locally in $B_{r_{*}}^{*}$ to a $u_{0}$ satisfying $\Delta u_{0}=f\left(u_{0}\right)$ in $B_{r_{*}}^{*}$. Otherwise, using (3-2),

$$
u_{0}(r) \geq G^{-1}\left(\frac{r^{2}}{2 N}\right) \quad \text { for } r<r_{*} .
$$

This estimate leads to $f\left(u_{0}\right) \in L^{1}\left(B_{1}\right)$, as in the end of (3-3). It is not difficult to see that $u_{0}$ is a weak solution of (1-1) in $B_{r_{*}}$. Moreover, noting that the estimate (3-3) is valid for any $t \leq t_{1}$, we get by passing $\alpha$ to 0 that

$$
u_{0}(r) \leq \frac{N}{N-2} G^{-1}\left(\frac{r^{2}}{2 N}\right) \text { for all } r \leq r_{*} .
$$

This ensures that $\lim _{r \rightarrow 0^{+}} u_{0}(r)=0$. It remains to prove that $u_{0}$ exists over all of $\mathbb{R}_{+}$. First, we can repeat the argument in the proof of Equation (2-1) to get a first turning point $r^{1}$ for $u_{0}$, and we find $u_{0}(r) \leq u_{0}\left(r^{1}\right)$ whenever the solution exists. It remains to prove that $u_{0}$ cannot reach 0 for any $r>0$. We distinguish two cases, first, that $\lim _{t \rightarrow 0^{+}} F(t)=-\infty$ and, second, that $\lim _{t \rightarrow 0^{+}} F(t) \in \mathbb{R}$.

For the first, because $-F\left(u_{0}(r)\right) \leq-F\left(u_{0}\left(r^{1}\right)\right)$ for $r \geq r^{1}$, we know $u_{0}$ cannot go near zero by the hypothesis on $F$, and so $u_{0}$ exists in all of $\mathbb{R}_{+}$.

For the second, define

$$
\bar{F}(t)=\int_{0}^{t} f(s) d s=F(t)+\int_{0}^{t_{0}} f(s) d s .
$$

Clearly (2-4) holds if we replace $F$ by $\bar{F}$. Doing this with $u_{\alpha}$ and passing the limit $\alpha \rightarrow 0$, we have

$$
\bar{F}\left(u_{0}\left(r^{1}\right)\right) \geq(N-1) \int_{0}^{r^{1}} \frac{u_{0}^{\prime}(s)^{2}}{s} d s>0 .
$$

Since $\bar{F}(0)=0$ and $\bar{F}\left(u_{0}(r)\right) \geq \bar{F}\left(u_{0}\left(r^{1}\right)\right)$ for $r \geq r^{1}$ by (2-4), we have $u_{0} \geq C>0$ for $r \geq r^{1}$, and hence $u_{0}$ is defined in $\mathbb{R}_{+}$.

Finally, as Lemma 2.2 and the proof of Theorem 2.4 are always valid, we obtain the desired oscillation and asymptotic behavior for any radial rupture solution, which completes our proof. 
Remark 3.2. We have rather precise behavior for the limiting solution near the origin, since

$$
G^{-1}\left(\frac{r^{2}}{2 N}\right) \leq u_{0}(r) \leq \frac{N}{N-2} G^{-1}\left(\frac{r^{2}}{2 N}\right) .
$$

But we don't know whether the radial singular solution is unique under the entire Condition 1.1. For some special cases, we will show some uniqueness result and more precise asymptotic behavior of $u_{0}$ at infinity in a forthcoming paper. Also, under the same assumptions of Proposition 2.5 or Corollary 2.6, the result holds for $\beta=0$ or $\gamma=0$, respectively.

By the proof, we observe also that Condition 1.1(ii) is sufficient to ensure the existence of a rupture solution of (1-1) in a small ball. Here we can even erase the monotonicity condition in Condition 1.1(ii) to get "almost" a local rupture solution of (1-1). In [Li et al. 2005], a positive rupture solution has been constructed in small balls under assumption that $(q-1) H(t) \leq t f(t)$ for $0 \leq t \leq A$, where $q>1$, $H(A)>0$, and $H(t)=\int_{t}^{\infty} f(s) d s$. As indicated in [Li et al. 2005], this condition guarantees that $\lim _{t \rightarrow 0^{+}} f(t)=\infty$ and essentially holds for nonlinearities such as $f(t)=t^{-q}$ with $q>1$. Here we prove a much more general result.

Theorem 3.3. Let $f$ be continuous in $\left(0, \tilde{t}_{0}\right]$ such that $\lim _{t \rightarrow 0^{+}} f(t)=\infty$ and

$$
\lim _{t \rightarrow 0^{+}} \frac{\widetilde{F}(t)}{f(t)}=0, \quad \text { where } \widetilde{F}(t)=\int_{t}^{\tilde{t}_{0}} f(s) d s .
$$

Then there exists an $r>0$ such that there is a radial function $u \in C\left(\bar{B}_{r}\right)$ satisfying $u(0)=0$ and $\Delta u=f(u)$ in $B_{r}^{*} \subset \mathbb{R}^{N}$.

Proof. Taking sufficiently small $\tilde{t}_{0}>0$, we can assume that $f$ is positive in $\left(0, \tilde{t}_{0}\right]$. For any $0<\Lambda \leq \tilde{t}_{0}$ and $\alpha \in(0, \Lambda]$, define $r_{\Lambda}=\inf \left\{t>0, u_{\alpha}(t)=\Lambda\right\}$, where $u_{\alpha}$ is the solution of (2-1). We see that such $r_{\Lambda}$ is well defined, since $u_{\alpha}$ is increasing in the region before $\tilde{t}_{0}$. The equality (3-1) implies that $r^{N-1} u_{\alpha}^{\prime}(r) \geq(C / N) r^{N}$ for $r \leq r_{\Lambda}$, where $C=\min _{\left(0, \tilde{t}_{0}\right]} f(t)>0$. Thus

$$
r_{\Lambda}^{2} \leq \frac{2 N}{C}(\Lambda-\alpha) \leq \frac{2 N \Lambda}{C} \text { for any } 0<\alpha \leq \Lambda \leq \tilde{t}_{0} .
$$

On the other hand, there exists a $C^{1}$ nondecreasing function $g(t)$ such that $\widetilde{F}(t) \leq g(t) f(t)$ in $\left(0, \tilde{t}_{0}\right]$ and $\lim _{t \rightarrow 0^{+}} g(t)=0$. Let

$$
H_{\alpha}(r)=r^{N}\left(\frac{u_{\alpha}^{\prime 2}}{2}+\widetilde{F}\left(u_{\alpha}\right)-\frac{N}{r} g\left(u_{\alpha}\right) u_{\alpha}^{\prime}\right) \quad \text { for } r \in\left[0, r_{\tilde{t}_{0}}\right] .
$$

A direct calculation then yields

$$
H_{\alpha}^{\prime}(r)=r^{N-1}\left[N \widetilde{F}\left(u_{\alpha}\right)-N g\left(u_{\alpha}\right) f\left(u_{\alpha}\right)-N g^{\prime}\left(u_{\alpha}\right) u_{\alpha}^{\prime 2}+\left(1-\frac{1}{2} N\right) u_{\alpha}^{\prime 2}\right] \leq 0,
$$


and thus $H_{\alpha}(r)$ is nonincreasing in $\left[0, r_{\tilde{t}_{0}}\right]$. So we get

$$
\frac{u_{\alpha}^{\prime 2}}{2}+\widetilde{F}\left(u_{\alpha}\right)-\frac{N}{r} g\left(u_{\alpha}\right) u_{\alpha}^{\prime} \leq 0 \quad \text { in }\left[0, r_{\tilde{t}_{0}}\right]
$$

which implies $u_{\alpha}^{\prime}(r) \leq 2 N g\left(u_{\alpha}\right) / r$ in $\left[0, r_{\tilde{t}_{0}}\right]$, since $\widetilde{F} \geq 0$ in $\left(0, \tilde{t}_{0}\right]$. We claim there then exists $r_{*}>0$ such that $r_{\tilde{t}_{0}}(\alpha) \geq r_{*}$ for any $\alpha>0$ small enough. To prove this, consider $v$ as the radial solution of

$$
v^{\prime \prime}+\frac{N-1}{r} v^{\prime}=C_{\Lambda}=\max _{\left[\Lambda, \tilde{t}_{0}\right]} f(t)
$$

with $v\left(r_{\Lambda}\right)=u_{\alpha}\left(r_{\Lambda}\right)=\Lambda$ and $v^{\prime}\left(r_{\Lambda}\right)=u_{\alpha}^{\prime}\left(r_{\Lambda}\right)>0$. It is not difficult to see that $v \geq u_{\alpha}$ for $r \in\left[r_{\Lambda}, r_{\tilde{t}_{0}}\right]$ and $\alpha<\Lambda$. Hence, for such $r$,

$$
\begin{aligned}
u_{\alpha}(r) \leq v(r)=\Lambda+\frac{1}{N-2}\left(u_{\alpha}^{\prime}\left(r_{\Lambda}\right) r_{\Lambda}-\frac{C_{\Lambda} r_{\Lambda}^{2}}{N}\right)\left(1-\frac{r^{2-N}}{r_{\Lambda}^{2-N}}\right) \\
+\frac{C_{\Lambda}\left(r^{2}-r_{\Lambda}^{2}\right)}{2 N} \\
\leq \Lambda+\frac{u_{\alpha}^{\prime}\left(r_{\Lambda}\right) r_{\Lambda}}{N-2}+\frac{C_{\Lambda} r^{2}}{2 N} \leq \Lambda+\frac{2 N g(\Lambda)}{N-2}+\frac{C_{\Lambda} r^{2}}{2 N} .
\end{aligned}
$$

Fixing $\Lambda_{0}>0$ small enough so that

$$
\Lambda_{0}+\frac{2 N g\left(\Lambda_{0}\right)}{N-2} \leq \frac{\tilde{t}_{0}}{2}
$$

By taking $r=r_{\tilde{t}_{0}}$ in (3-9), we obtain $r_{\tilde{t}_{0}}^{2} \geq N \tilde{t}_{0} / C_{\Lambda_{0}}$ for $\alpha \leq \Lambda_{0}$; so our claim is true.

Combining this with the estimate (3-8), we have $u_{\alpha}(r) \in\left[C \delta^{2} / 2 N, \tilde{t}_{0}\right]$ uniformly for any $0<\alpha<\delta<\min \left(\Lambda_{0}, r_{*}\right)$ and $r \in\left[\delta, r_{*}\right]$. Using standard elliptic theory and the diagonal process, we get a subsequence such that $u_{\alpha_{n}}$ converges in $C^{1}$ locally in $B_{r_{*}}^{*}$ to a $u_{0}$ satisfying $\Delta u_{0}=f\left(u_{0}\right)$ in $B_{r_{*}}^{*}$.

On the other hand, since $u_{\alpha}(r) \leq \Lambda$ in $\left[0, r_{\Lambda}\right]$, we may combine this with (3-9) and conclude that for all $0<\alpha \leq \Lambda \leq \Lambda_{0}$, we have

$$
u_{\alpha}(r) \leq \Lambda+\frac{2 N g(\Lambda)}{N-2}+\frac{C_{\Lambda} r^{2}}{2 N} \text { in }\left[0, r_{*}\right]
$$

Passing the limit $\alpha \rightarrow 0^{+}$, we find the same estimate holds for $u_{0}$. Recalling that $\lim _{t \rightarrow 0^{+}} g(t)=0$, we can hence choose for any $\epsilon>0$ an $\Lambda \in(0, \epsilon)$ such that $g(\Lambda) \leq \epsilon$. By (3-10) with now $\alpha=0$, we have $u_{0}(r) \leq 8 \epsilon$ for $r$ sufficiently small; this then yields $\lim _{r \rightarrow 0} u_{0}(r)=0$. 
Remark 3.4. The difference between Theorems 3.1 and 3.3 is that in the first case, Condition 1.1 ensures the existence of a entire weak solution, while this is not always the case for the second one.

\section{An application}

One direct consequence of Theorems 2.1 and 3.1 is the structure of radial solutions to the eigenvalue Neumann problem

$$
\Delta u=\lambda f(u) \quad \text { in } B \subset \mathbb{R}^{N}, \quad \frac{\partial u}{\partial v}=0 \quad \text { on } \partial B,
$$

where $f$ satisfies Condition $1.1, N \geq 3$, and $\lambda>0$.

Theorem 4.1. Let $\alpha^{*}$ be defined as in Theorem 2.9. For any $0 \leq \alpha<\alpha^{*}$, there is an increasing sequence $\left\{\lambda_{n}(\alpha)\right\}$ such that for $\lambda=\lambda_{n}$, (4-1) has a radial solution $u_{n}(r)$ satisfying $u_{n}(0)=\alpha$. Moreover, the function $u_{n}-t_{0}$ has exactly $n$ zeros for $r \in(0,1)$. Meanwhile, if $\alpha<\alpha^{*}$ and $\lambda \notin\left\{\lambda_{n}(\alpha)\right\}$, then (4-1) has no radial solution $u$ satisfying $u(0)=\alpha$.

Proof. Theorems 2.1, 2.9, and 3.1 provide an entire oscillate radial solution such that $u(0)=\alpha$ for any $0 \leq \alpha<\alpha^{*}$.

Using the transformation $w(x)=u_{\alpha}(\sqrt{\lambda} x)$, it is easy to see that $\Delta w=\lambda f(w)$ in $B$ and $\partial_{\nu} w(x)=\sqrt{\lambda} u_{\alpha}^{\prime}(\sqrt{\lambda})$ on $\partial B$. Thus for $\lambda_{n}=\left(r_{\alpha}^{n}\right)^{2}$ with $\left\{r_{\alpha}^{n}\right\}$ the increasing sequence of positive critical points of $u_{\alpha}$, we get a solution of (4-1).

On the other hand, if $u$ is a radial solution of (4-1) with $\alpha>0$, the function $v(x)=u(\sqrt{\lambda} x)$ satisfies the initial value problem (2-1), and so it coincides with the unique solution $u_{\alpha}$. This means that we must have $u^{\prime}(\sqrt{\lambda})=0$, that is, there exists an $n$ such that $\lambda=\left(r_{\alpha}^{n}\right)^{2}$.

\section{Appendix: Cases $N=1$ and 2}

Here we show that the situation is quite different between the dimensions $N \leq 2$ and $N \geq 3$. Consider Equation (1-3) with $f$ satisfying Condition 1.1. For $N=1$, we have the following result.

Proposition A.1. If $u^{\prime \prime}=f(u)$ with $u(0)=\alpha \in\left(0, t_{0}\right)$ and $u^{\prime}(0)=0$, then the solution will be globally defined in $\mathbb{R}_{+}$, and either $u$ is periodic, or $u$ is increasing and $\lim _{r \rightarrow \infty} u(r)=\infty$.

Proof. Letting $F$ be the primitive of $f$ defined by (2-3), we then have

$$
\frac{1}{2} u^{\prime}(r)^{2}-F(u(r))=-F(\alpha) .
$$

By the variation of $F$, there exists at most one point $\beta>t_{0}$ such that $F(\beta)=F(\alpha)$. Hence $u^{\prime}(r)=0$ if and only if $u(r)=\alpha$ or $u(r)=\beta$. Since $u$ is increasing near 0 , 
we have two possibilities: either $u$ remains increasing or there exists an $r_{0}$ such that $u\left(r_{0}\right)=\beta$. In the first case, $u^{\prime}(r)$ is uniformly bounded since $F \leq 0$; this yields that $u$ is globally defined in $\mathbb{R}_{+}$, and we conclude $\lim _{r \rightarrow \infty} u(r)=\gamma$ exists. As in the proof of Theorem 2.1, we must have $\gamma=\infty$, since the only zero of $f$ satisfies $f^{\prime}\left(t_{0}\right)<0$. In the second case, by extending as $u(r)=u\left(2 r_{0}-r\right)$ on $\left[r_{0}, 2 r_{0}\right]$ and so on, we obtain a periodic solution.

We should mention that when $N=1$, Laugesen and Pugh [2000b] have considered very carefully the properties of positive periodic solutions or rupture solutions for thin film equations. We next have the situation in $\mathbb{R}^{2}$ :

Proposition A.2. Let $N=2$. For any $\alpha \in\left(0, t_{0}\right)$, the solution $u_{\alpha}$ to (1-3) is always globally defined on $\mathbb{R}_{+}$. Either $u_{\alpha}$ oscillates around $t_{0}$ with $\lim _{r \rightarrow \infty} u_{\alpha}(r)=t_{0}$, or $u_{\alpha}$ is increasing and $\lim _{r \rightarrow \infty} u_{\alpha}(r)=\infty$.

Proof. In fact, either $u$ is increasing, and we can say that $0 \leq u^{\prime} \leq C$, and hence $u$ exists globally and must satisfy $\lim _{r \rightarrow \infty} u_{\alpha}(r)=\infty$; or there exists $r^{1}$ such that $u^{\prime}\left(r^{1}\right)=0$ and $u^{\prime \prime}\left(r^{1}\right)<0$. Then the rest of the proof of Theorem 2.1 and 2.4 holds when $N=2$.

All situations can occur. If $\lim _{t \rightarrow \infty} F(t)=-\infty$, we see by $F(u(r)) \geq F(\alpha)$ that $u$ cannot tend to $\infty$, and so $u$ must oscillate around $t_{0}$. An example is when $f(u)=u^{-p}-C$ with $p, C>0$.

On the other hand, we may consider the case $\lim _{t \rightarrow \infty} F(t)<\lim _{t \rightarrow 0^{+}} F(t)$ when $N=1$. Taking $\alpha>0$ small enough so that there is no $\beta>\alpha$ satisfying $F(\beta)=F(\alpha)$, we then obtain $\lim _{r \rightarrow \infty} u_{\alpha}(r)=\infty$. For $N=2$, we show in the following some examples in which $\lim _{r \rightarrow \infty} u(r)=\infty$.

Let $s_{1}>0$. Let $g$ be a $C^{1}$ function on $\mathbb{R}_{+}$for which $g>0$ on $\left[0, s_{1}\right), g^{\prime}\left(s_{1}\right)<0$, $g$ is constant near $0, g<0$ on $\left(s_{1}, \infty\right)$, and

$$
\int_{0}^{\infty} s g(s) d s=\gamma>0 \text { is convergent. }
$$

Define

$$
u_{\alpha}(r)=\alpha+\int_{0}^{r} \frac{1}{s} \int_{0}^{s} \sigma g(\sigma) d \sigma d s \quad \text { for } \alpha>0 .
$$

Of course, $u_{\alpha}$ is increasing from $\alpha$ to $\infty$. Let $f(t)=g \circ u_{\alpha}^{-1}(t)$ be defined on $[\alpha, \infty)$. It is clear that $f$ is $C^{1}$ on $[\alpha, \infty)$ and that $f$ has a single zero $t_{0}=u_{\alpha}\left(s_{1}\right)$ with $f^{\prime}\left(t_{0}\right)<0$. We need only to extend $f$ on $(0, \alpha)$ by positive values to satisfy Condition 1.1(ii). We obtain $\Delta u_{\alpha}=f\left(u_{\alpha}\right)$ in $\mathbb{R}^{2}$ with $u_{\alpha}(0)=\alpha<t_{0}$. 


\section{Acknowledgments}

Part of this work was completed while Zhou was visiting the University of CergyPontoise, and he thanks the Department of Mathematics for its warm hospitality. The authors thank the referee for valuable comments.

\section{References}

[Bertozzi and Pugh 1998] A. L. Bertozzi and M. C. Pugh, "Long-wave instabilities and saturation in thin film equations", Comm. Pure Appl. Math. 51:6 (1998), 625-661. MR 98m:35171 Zbl 0916.35008

[Bertozzi and Pugh 2000] A. L. Bertozzi and M. C. Pugh, "Finite-time blow-up of solutions of some long-wave unstable thin film equations", Indiana Univ. Math. J. 49:4 (2000), 1323-1366. MR 2002d:35030 Zbl 0978.35007

[Bertozzi et al. 2001] A. L. Bertozzi, G. Grün, and T. P. Witelski, "Dewetting films: bifurcations and concentrations", Nonlinearity 14:6 (2001), 1569-1592. MR 2003m:76007 Zbl 1006.35049

[Burelbach et al. 1988] J. P. Burelbach, S. G. Bankoff, and S. H. Davis, "Nonlinear stability of evaporating/condensing liquid films”, J. Fluid Mech. 195 (1988), 463-494.

[Grün 2004] G. Grün, "Droplet spreading under weak slippage - existence for the Cauchy problem”, Comm. Partial Differential Equations 29:11-12 (2004), 1697-1744. MR 2005i:35109 Zbl 02171338

[Guo and Wei 2007] Z. Guo and J. Wei, "Symmetry of nonnegative solutions of a semilinear elliptic equation with singular nonlinearity", Proc. Roy. Soc. Edinburgh Sect. A 137:5 (2007), 963-994. MR 2359923 Zbl 05235749

[Guo et al. 2006] H. Guo, Z. Guo, and K. Li, "Positive solutions of a semilinear elliptic equation with singular nonlinearity”, J. Math. Anal. Appl. 323:1 (2006), 344-359. MR 2007f:35090 Zbl 05072948

[Hwang et al. 1997] C. Hwang, C. Lin, and W. Uen, "A nonlinear three-dimensional rupture theory of thin liquid films", J. Colloid Interf. Sci. 190 (1997), 250-252.

[Jiang and Lin 2004] H. Jiang and F. Lin, "Zero set of Sobolev functions with negative power of integrability”, Chinese Ann. Math. Ser. B 25:1 (2004), 65-72. MR 2004m:46071 Zbl 02066972

[Jiang and Ni 2007] H. Jiang and W.-M. Ni, "On steady states of van der Waals force driven thin film equations”, European J. Appl. Math. 18:2 (2007), 153-180. MR 2008c:35077 Zbl 05206846

[Jones and Küpper 1986] C. Jones and T. Küpper, "On the infinitely many solutions of a semilinear elliptic equation”, SIAM J. Math. Anal. 17:4 (1986), 803-835. MR 87h:35261 Zbl 0606.35032

[Joseph and Lundgren 1972/73] D. D. Joseph and T. S. Lundgren, "Quasilinear Dirichlet problems driven by positive sources”, Arch. Rational Mech. Anal. 49 (1972/73), 241-269. MR 49 \#5452 Zbl 0266.34021

[Laugesen and Pugh 2000a] R. S. Laugesen and M. C. Pugh, "Linear stability of steady states for thin film and Cahn-Hilliard type equations", Arch. Ration. Mech. Anal. 154:1 (2000), 3-51. MR 2002k:35056 Zbl 0980.35030

[Laugesen and Pugh 2000b] R. S. Laugesen and M. C. Pugh, "Properties of steady states for thin film equations", European J. Appl. Math. 11:3 (2000), 293-351. MR 2002g:76007 Zbl 1041.76008

[Li et al. 2005] K. Li, H. Guo, and Z. Guo, "Positive single rupture solutions to a semilinear elliptic equation”, Appl. Math. Lett. 18:10 (2005), 1177-1183. MR 2006b:35105 Zbl 05001430 
[Slepčev and Pugh 2005] D. Slepčev and M. C. Pugh, "Selfsimilar blowup of unstable thin-film equations", Indiana Univ. Math. J. 54:6 (2005), 1697-1738. MR 2007a:35130 Zbl 05014746

Received May 11, 2007. Revised September 27, 2007.

\section{ZONGMING GUO}

DEPARTMENT OF MATHEMATICS

HENAN NORMAL UNIVERSITY

XINXIANG 453002

CHINA

guozm@public.xxptt.ha.cn

DONG YE

DÉPARTEMENT DE MATHÉMATIQUES, UMR 8088

Université de CERGY-PontoIse

Site SAint-Martin, BP 222

95302 CERgy-Pontoise CedeX

FRANCE

dong.ye@u-cergy.fr

FENG ZHOU

DEPARTMENT OF MATHEMATICS

EAST CHINA NORMAL UNIVERSITY

SHANGHAI 200062

CHINA

fzhou@math.ecnu.edu.cn 